Service social

\title{
Virage néolibéral, virage patriarcal. Une entrevue avec Lucie Bélanger de Relais-Femmes
}

\section{Michèle Clément}

Volume 44, numéro 2, 1995

Visages de la violence

URI : https://id.erudit.org/iderudit/706690ar

DOI : https://doi.org/10.7202/706690ar

Aller au sommaire du numéro

Éditeur(s)

École de service social de l'Université Laval

ISSN

1708-1734 (numérique)

Découvrir la revue

Citer ce document

Clément, M. (1995). Virage néolibéral, virage patriarcal. Une entrevue avec

Lucie Bélanger de Relais-Femmes. Service social, 44(2), 9-21.

https://doi.org/10.7202/706690ar d'utilisation que vous pouvez consulter en ligne.

https://apropos.erudit.org/fr/usagers/politique-dutilisation/ 


\section{E N T R R E V U E}

\section{Virage néolibéral, virage patriarcal Une entrevue avec Lucie Bélanger de Relais-Femmes}

MICHÈLE CLÉMENT

Centre de recherche interdisciplinaire sur la violence familiale et la violence faite aux femmes (CRI-VIFF)

Lucie Bélanger a obtenu sa maîtrise en sociologie de I'Université du Québec à Montréal en 1989. Militante de cœur et de choix, elle s'est très tôt engagée dans la cause des femmes et la défense des droits socio-économiques pour tous. Elle travaille depuis 1992 à Relais-Femmes comme agente de développement et est membre active du CRI-VIFF depuis sa fondation.

Lucie, on parle souvent de la violence comme d'une situation qui relève de la vie privée ou de l'intimité des gens. Dans une de vos conférences, je vous ai cependant entendu parler de l'impact des changements dans les politiques sociales sur la vulnérabilité des femmes à la violence. J'aimerais que vous me parliez un peu de cette conjoncture dans laquelle surviennent les changements de politiques sociales. 
Pour commencer, j'aimerais revenir sur le virage que nous avons vécu au cours des dernières années. Lorsque j'ai commencé à travailler en milieux communautaires, comme formatrice auprès de gens venant de syndicats ou de groupes d'actions catholiques ouvrières, nous avions l'habitude d'ouvrir chacune des sessions de formation par un tour de table. Les gens parlaient alors de leur droit au travail. C'était en 1980. Cinq ans plus tard, lorsque j'ai quitté le Centre pastoral en milieu ouvrier (CPMO), on ne parlait déjà plus, dans les tours de table, du droit au travail, mais plutôt du privilège de travailler. Ce changement dans le discours témoigne bien, selon moi, du virage fondamental qu'a connu notre société.

Nos gouvernements et leurs dirigeants ne se donnent plus comme autrefois la responsabilité de garantir les conditions nécessaires à l'exercice des droits reconnus. Tout au plus assurent-ils le minimum vital. La logique du dernier recours redevient le principe structurant des politiques sociales. Or, substituer la notion de besoins minimaux à celle de droit est un recul très important. En fait, l'État-providence s'effrite actuellement de tous les côtés. Il fut une époque, cependant, où ce même État a été le moteur de la création d'espaces démocratiques pour les femmes.

Les analyses que I'on fait aujourd'hui de l'État-providence ont presque toujours pour ancrage l'idée que les gens en ont abusé. On parle beaucoup de la nécessité de revenir à une démocratie plus contrôlée, plus disciplinée parce que, semble-t-il, on a créé une catégorie de gens dépendants qui attendent tout de tout le monde. Lorsque j'entends de tels propos, je me demande sur quelle planète j'ai vécu, car ce n'est pas du tout l'analyse que je fais des conséquences de la mise en œuvre de l'État-providence. Et, plus que d'autres peutêtre, les femmes ont fait durant cette période d'immenses gains. Je m'explique.

Prenons par exemple la Loi sur l'aide sociale. Jusqu'à I'avènement de cette loi, l'État assurait une certaine aide aux mères nécessiteuses. Qui étaient ces femmes? Des mères abandonnées par leur conjoint et qui devaient subvenir seules aux besoins de jeunes enfants. Il faut faire attention de ne pas confondre les mères nécessiteuses avec les femmes qui avaient droit à une pension de veuve à la suite du décès ou de la maladie du conjoint. Les mères nécessiteuses étaient plutôt des femmes qui se retrouvaient seules en raison de I'abandon du conjoint.

Selon les normes de l'époque, ces dernières recevaient de l'État un certain montant d'argent pour subvenir aux besoins de leurs enfants. Jusqu'ici tout va bien. La situation pour elles se compliquait 
toutefois dans la mesure où, pour encaisser ce chèque, elles devaient nécessairement le faire contresigner par un notable. Pourquoi ? Pour témoigner sans plus de leur moralité et de leurs bonnes mœurs. On aura compris que le notable n'était nul autre alors que le notaire, le médecin ou le marchand de la place.

La création de la Loi sur I'aide sociale viendra modifier cette situation. À la moralité, approuvée sur la base d'un jugement d'homme, on substituera le critère beaucoup plus objectif de l'écart entre les ressources et les besoins donnant droit à des prestations. De l'État gardien de la moralité des femmes, on passe à un État redistributeur avec une connotation de substitut pourvoyeur. La Loi sur I'aide sociale basée sur un droit, qui n'est qu'un des nombreux acquis de la mise en place de l'État-providence, a ainsi conféré aux femmes un statut de citoyennes à part entière. Dans les analyses que I'on fait aujourd'hui de cette période, on ne doit pas sous-estimer l'importance de ce fait.

À la même époque, une autre loi allait être très importante pour les femmes : la Loi sur le divorce. Combinée à la Loi sur I'aide sociale, cette dernière allait en effet devenir un outil juridique de taille pour que les femmes s'affranchissent de situations telles que la violence conjugale. D'autres motifs justifiaient aussi le divorce. Ce qui est sûr, cependant, c'est que dans tous les cas la Loi sur l'aide sociale donnait l'accès aux femmes à des ressources économiques leur permettant d'exercer leur droit politique (Loi sur le divorce).

Pour toutes ces raisons, je suis incapable de faire une lecture de ces politiques comme étant créatrices de dépendance chez les femmes ou, dans une mesure plus large, dans d'autres strates de la population. Il faut au contraire se resituer dans le contexte de l'époque pour apprécier jusqu'à quel point ces lois ont été bénéfiques pour les femmes.

\section{Ce que vous dites, finalement, c'est que les lois relatives à l'aide sociale et au divorce ont été des outils extrêmement importants pour l'affranchissement des femmes. Mais en quoi ces lois sont-elles si différentes aujourd'hui?}

Je dois dire, d'abord, qu'outre les gains observables à un premier niveau, ces lois possédaient aussi un contenu implicite sur lequel je me dois de revenir un peu. Il est très intéressant de voir, en effet, qu'à ses débuts la loi sur l'aide sociale prévoyait des montants de base établis selon les besoins particuliers de chaque femme (ex. : nombre 
d'enfants). Il y avait aussi des montants réservés à des besoins spéciaux, tels que la réparation d'appareils ménagers, l'achat d'une literie, etc. En acceptant l'existence de tels besoins, l'État se mettait en position d'agir envers les femmes nécessiteuses de la même façon que l'aurait fait $n$ 'importe quel honnête père pourvoyant aux besoins de sa famille. Mais, plus encore, c'est la production sociale non salariée des femmes qu'on rendait visible à travers la reconnaissance de ces besoins spéciaux. Plutôt que de remplir les orphelinats, on a choisi de laisser aux femmes leur rôle d'éducatrice des enfants en leur rendant cette situation financièrement possible.

L'avénement de la Loi sur l'aide sociale repose pourtant sur un schéma patriarcal dans la mesure où l'État continue de considérer que la situation normale pour une femme est de vivre des subsides d'un pourvoyeur auquel il accepte de se substituer bien volontiers, mais toujours dans une perspective temporaire. La réforme de l'aide sociale, dans les années 80 , a d'ailleurs bien mis en valeur cet aspect de la loi. Dans un rapport confidentiel du Conseil des ministres, il est clairement dit que les femmes et les jeunes - parce qu'à cette époque commençait déjà à poindre le problème majeur du non-accès à l'emploi pour les jeunes - avaient détourné l'aide sociale de son véritable objectif, soit le dernier recours. En d'autres mots, ce qui était sous-entendu dans ce rapport, du moins en ce qui concerne les femmes, c'est que l'aide sociale devait être un soutien transitoire et que le chemin, le "vrai chemin », était soit l'accès à l'emploi salarié, soit le retour à un conjoint ou, idéalement, la combinaison de ces deux solutions.

Quoi qu'il en soit, un fait est certain: la Loi sur l'aide sociale reconnaît, implicitement, que pour élever des enfants il est nécessaire d'avoir un revenu garanti. À travers cette loi, les femmes ont donc pu expérimenter l'importance de leur travail auprès des enfants et tout cela en ayant la possibilité d'exercer ce travail autrement que dans un rapport de dépendance économique au conjoint. Combien de femmes m'ont dit: "Écoute, depuis que je suis à l'aide sociale, c'est vrai que je me retrouve plus pauvre, mais c'est moi qui décide ce que je fais avec le chèque, c'est moi qui paie le loyer, c'est moi qui vois aux soins des enfants. »

À mon avis, nous n'avons pas assez regardé ce qu'a signifié pour les femmes l'arrivée d'une loi comme celle de l'aide sociale et j'ai très peur lorsque j'entends les gens en parler comme s'il s'agissait d'une loi qui crée de la dépendance et de l'oisiveté. La Loi sur l'aide sociale, au contraire, a permis de rendre visible le voile qui pesait jusque-là entre la vie amoureuse et la survie économique des femmes. 
La réflexion du mouvement féministe au Québec sur la valeur sociale du travail ménager et sur sa non-rémunération s'articule étroitement avec l'avènement de cette loi.

La mise en place de l'État-providence a donc été capitale pour les femmes, et ne pas reconnaître ce fait biaise profondément le sens de nos analyses. Pensons, par exemple, au développement qu'elle a assuré dans le secteur de la santé et des services sociaux. Qu'a permis cette extension des services? La reconnaissance des compétences historiquement dévolues aux femmes et, par conséquent, la possibilité de faire entrer sur le terrain du travail salarié ce qui, traditionnellement, a été vu comme une fonction naturelle des femmes. La mise en place de l'État-providence a permis aux femmes de pénétrer le marché du travail, parce que les emplois qui ont été créés dans ce contexte faisaient principalement appel à leurs compétences naturelles (soins, éducation, etc.).

De façon moins visible, elle a aussi permis de diminuer leurs responsabilités familiales. La mise sur pied d'un réseau de garderies et l'accessibilité, en dehors de la sphère privée, à des soins de santé ont été sans conteste des acquis qui ont largement contribué à diminuer leur fardeau au sein de la famille.

Le développement de logements sociaux (HLM, coops d'habitation) a aussi connu à cette époque un essor considérable. Ce type d'habitation était vu, au départ, comme une rémunération différée permettant de bonifier soit les bas salaires, soit les revenus modestes de l'aide sociale. Sur le plan de la stabilisation économique et émotive des femmes en situation de vulnérabilité (familles monoparentales, séparation pour motifs de violence conjugale, etc.), le développement du logement social a donc été un autre élément très important. Le fait pour ces femmes de disposer d'un loyer dont le coût ne risquait pas à chaque mois de monter en flèche était très rassurant.

Je pourrais donner bien d'autres exemples encore des acquis réalisés par les femmes et par la population en général à travers la mise en place de l'État-providence. Je ne voudrais pas laisser entendre, ici, qu'il faut revenir à ce qui était, point à la ligne. Les choses ont évidemment changé. Mais faisons attention de ne pas jeter le bébé avec l'eau du bain. On ne doit pas sous-estimer, sur le plan de nos analyses, ce qu'a signifié et ce que continue de signifier l'arrivée de l'État-providence, car c'est cet État qui s'est donné la responsabilité de permettre de facto la réalisation des droits sociaux et économiques des personnes, notamment des femmes.

Avec le virage néolibéral, I'État a laissé tomber son mandat de démocratisation des droits socio-économiques. Ces droits sont 
maintenant relégués à la sphère du privé. Concrètement, cet État ne se sent plus concerné que par les besoins minimaux des individus. Personnellement, j'endosse entièrement l'analyse faite par les féministes américaines et scandinaves qui considèrent qu'à travers la venue et l'enracinement du néolibéralisme, c'est tout le patriarcat qui revient en force. Bien sûr, il se fait beaucoup moins visible et présent dans la sphère privée, mais on le retrouve néanmoins en germe à travers les lois, les règlements et les orientations économiques.

\section{Comment peut-on réorganiser le patriarcat à travers une loi ou un règlement? Avez-vous un exemple permettant d'illustrer ce phéno- mène?}

Les réaménagements de la Loi sur l'aide sociale sont éclairants à ce sujet. L'opération boubou-macoute ciblait très clairement les femmes, et les femmes dans leurs rapports affectifs essentiellement. En d'autres mots, l'opération consistait à dépister celles qui entretenaient des relations affectives avec un homme et ce, afin de pouvoir les déclarer en situation de vie maritale. Ce que I'on cherchait, en fait, $c^{\prime}$ était d'obliger les femmes à revenir à une situation de dépendance économique vis-à-vis des hommes. Une agente d'aide sociale, sous le couvert de l'anonymat, m'a expliqué à l'époque une des façons dont on s'y prenait pour mettre le grappin sur ces femmes. On sortait au hasard, par exemple, toutes les femmes chefs de famille payant un loyer de plus de 400 \$ par mois. Une mensualité de cet ordre était vue, on l'aura compris, comme un indice sérieux d'un accès à d'autres sources de revenu que la prestation de l'aide sociale.

Cette façon de procéder en dit très long sur les véritables droits des femmes à avoir accès à des ressources économiques sur une base autonome et en dehors d'un revenu décent ou de la dépendance affective envers un homme. La toile de fond de la réforme de l'aide sociale, du moins en ce qui concerne les femmes, est le contrôle, et le contrôle est une dimension inhérente au patriarcat.

Cette tendance, selon toute vraisemblance, risque d'ailleurs d'être accentuée dans les prochains mois. La rumeur veut en effet que les réformes de I'aide sociale à l'agenda des gouvernements fassent sauter ce qu'on peut considérer comme un des seuls acquis réchappés lors de la dernière réforme, soit la non-contrainte à l'emploi pour les femmes ayant un enfant de moins de six ans. En s'attaquant à cet acquis, ce qu'on vient affirmer en quelque sorte est que la réinsertion, le vrai retour, c'est l'emploi... Mais, plus subtilement encore, ce que I'on dit à travers cette réforme $c^{\prime}$ est que la production sociale des 
femmes en dehors du marché de l'emploi n'est pas une production sociale, que la sphère domestique n'est pas un lieu reconnu pour la réinsertion. À cet exemple, on voit bien l'important recul qui s'effectue à travers le virage néolibéral, non seulement sur le plan des droits sociaux mais aussi sur celui des idées.

L'emploi salarié est bien sûr un chemin privilégié pour l'accès à l'autonomie économique des femmes. Ce chemin reste toutefois piégé si on ne reconnaît pas en même temps l'importance du travail des femmes en dehors du marché à l'emploi. L'importante question de l'heure concernant la conciliation travail-famille témoigne bien de cette solidarité entre les deux sphères de la vie.

Avec l'incrustation en force du néolibéralisme, on cherche au fond à nous ramener à un capitalisme pur et dur où le marché est le maître absolu. Or, je ne comprends pas que l'on puisse adhérer à I'idée que le capitalisme pourrait répondre à l'utopie du plein emploi pour tous. Depuis les débuts du capitalisme, il a fallu intervenir sur les problèmes sociaux. D'abord par l'entremise de la charité publique, puis par le développement de politiques sociales. On essaie aujourd'hui de nous faire miroiter l'idée que le marché peut générer par lui-même des emplois et des revenus décents pour tous. Cette tentative $n^{\prime}$ est rien d'autre, au fond, qu'un retour à la vieille morale voulant que chacun soit responsable de soi et qu'avec un peu de courage on peut se trouver un emploi et un bon emploi. En bref, la voie royale de la réinsertion reste le travail salarié. Et, de là, s'enchaîne tout le reste, à savoir qu'avec un bon emploi il est possible d'avoir accès à un logement décent, à des soins de santé adéquats, etc.

Historiquement, les politiques sociales touchant l'habitation ont été d'un apport fondamental en matière de salaire différé. $C^{\prime}$ est ce type de politique qui a permis de bonifier et de qualifier un revenu qui, autrement, serait demeuré insuffisant. Aujourd'hui, on veut nous faire accepter l'idée qu'il est normal qu'une portion de $50 \%$ du salaire soit affectée au loyer, tant pour ceux qui vivent d'un revenu de transfert que pour ceux qui sont en emploi. Il n'en est rien, cependant, et cette situation s'avère d'autant plus tragique pour les femmes qui, comme on l'a vu lors de la marche Du pain et des roses, sont encore à revendiquer la hausse du salaire minimum, une loi sur l'équité salariale et l'élaboration d'un véritable programme d'infrastructure sociale.

Mais où sont ces emplois qui, pour emprunter l'expression de mon amie et inspiratrice Lucie Lamarche, professeure en droit, seraient à la fois qualifiés, qualifiant et qualifiés aux normes du droit? Ce que l'on est en train de faire avec la revendication des femmes 
pour un programme d'infrastructure sociale est plus qu'éloquent à ce sujet. Cette revendication, grandement inspirée de la réflexion faite par les services de garde en Ontario qui avaient interpellé monsieur Chrétien au moment des élections fédérales, se résume à peu près de la façon suivante: "Monsieur Chrétien, vous parlez d'un programme d'infrastructure créateur d'emplois, mais les emplois dont vous parlez sont des emplois d'hommes. Selon vous, les infrastructures dans une société ce sont les réseaux routiers et l'électricité. Mais nous, les femmes, notre travail dans le réseau communautaire, dans les garderies, dans les soins de santé, l'accompagnement que nous faisons auprès des personnes en difficulté, bref, tout ce qui relève du secteur communautaire, des groupes autonomes de femmes et de notre rôle au sein de la famille, tout cela ne fait aucunement partie de ce que vous appelez l'infrastructure nécessaire au maintien d'une société et de la qualité de vie dans cette société. Ce que nous voulons, c'est un programme d'infrastructure qui va créer de véritables emplois et des emplois dans les secteurs où sont actuellement concentrées les femmes."

De quelle façon le gouvernement a-t-il répondu à cette demande? Sous des dehors qui semblent légitimer ces revendications, il a plutôt tenté de profiter de l'occasion pour déployer des programmes d'employabilité viciés. Ces programmes ne sont rien d'autre, en réalité, que de légers suppléments de revenu. Ils ne répondent en rien à la revendication fondamentale des femmes portant cette cause, qui consiste à refuser d'accepter d'aller en dessous du seuil d'emploi réel soumis aux normes et au Code du travail.

Avec une telle revendication, les femmes touchent directement le nerf de la réorganisation des néolibéraux qui cherchent à utiliser les exclus du secteur de l'emploi et les personnes en situation d'extrême précarité (les personnes au chômage, les assistés sociaux) pour restructurer une main-d'œuvre qui ne serait plus soumise au droit du travail. C'est ainsi, par exemple, que dans le discours la notion d'irritants sociaux remplace maintenant celle de droits sociaux, les droits sociaux étant, bien entendu, le droit à la syndicalisation, le droit à un logement et à un niveau de vie décent.

Nous avons là, à mon avis, un bel exemple de la réorganisation du patriarcat. De la même façon que le capitalisme s'est développé et s'est structuré en s'appuyant sur la production non salariée, invisible et non reconnue des femmes, de la même façon on cherche actuellement, par l'entremise des politiques sociales, à exploiter les femmes par la gestion des espaces non productifs au quotidien et sur une trame de vie. Qui prend en charge ces personnes, sinon les 
femmes dans le privé et au sein des organismes communautaires? Et dans la vie, le temps de l'enfance, le temps de la maladie, le temps de la vieillesse, tous ces espaces qui ne sont pas ce qu'on appelle des espaces productifs, qui prend en charge ces personnes en situations de besoin, sinon les femmes?

Il faut aussi se demander si le modèle papa pourvoyeur et maman à la maison était aussi parfait qu'on a bien voulu nous le laisser croire. Ce modèle a-t-il vraiment fonctionné dans la logique même sous laquelle on nous le présente? Combien de femmes au Québec ont eu des chambreurs, ont fait de la couture, des ménages dans les maisons privées pour parvenir à boucler les fins de mois? Derrière le mythe de I'homme pourvoyeur se cachent des heures et des heures de travail non considérées. Alors, lorsque je vois l'État chercher à se déresponsabiliser sur le dos des femmes, je ne peux qu'être effrayée.

Prenons, par exemple, la question du virage ambulatoire. Plutôt que de rester deux semaines à I'hôpital, le malade $n^{\prime} y$ restera plus que trois jours. Mais qui va absorber les conséquences de ces changements, sinon les femmes à la maison et le mouvement communautaire?

Ce qui se passe actuellement avec les personnes âgées est un autre exemple frappant de l'érosion des politiques sociales et de la vulnérabilité que cela engendre chez les femmes. Au début des années 80, une étude fédérale a mis en évidence l'ampleur de la pauvreté chez les personnes âgées et plus particulièrement encore de la pauvreté des femmes âgées. Pour contrecarrer ce phénomène, le gouvernement a alors mis en place une politique $d^{\prime}$ 'indexation des pensions de vieillesse, ce qui s'est traduit, au fil des années, par une amélioration notable de leurs conditions économiques. Que se passe-t-il à l'heure actuelle? On voit apparaître des mesures remettant en question I'universalité des pensions de vieillesse pour y substituer la notion d'une rente tributaire du niveau de revenu familial. Qu'est-ce que cela veut dire pour beaucoup de femmes âgées? Rien d'autre que le retour vers une dépendance vis-à-vis du conjoint, le revenu des hommes étant toujours et encore nettement supérieur à celui des femmes.

Une qualité de vie fondée sur les droits reconnus par nos chartes ne relèverait donc plus des responsabilités des États provinciaux et fédéraux; ceux-ci ne se porteraient plus garants que des besoins minimaux. En d'autres mots, il ne s'agit plus d'aider les individus à vivre, mais plutôt de les empêcher de crever. Pour tout le reste, on se tourne vers la communauté... II se trouve cependant que si I'on supprime les outils collectifs pour atteindre cette qualité de vie, par exemple l'accès à des logements sociaux, on supprime du coup 
la possibilité réelle de respecter ces droits. Lorsqu'on a procédé à la réforme de l'aide sociale, on a vu s'opérer ce glissement. Avec la réforme, l'aide sociale a cessé d'être un droit basé sur l'écart réel entre les besoins et les ressources, mais est plutôt devenue une politique définie au regard de la non-aptitude ou de l'aptitude au travail.

$\mathrm{C}^{\prime}$ est ainsi qu'on revient à une logique du mérite, à la méritocratie, et cela dans un contexte où les emplois s'effondrent. On sait très bien que le problème n'est pas sur le plan de la formation professionnelle. On aurait beau renvoyer tout le monde dans les universités, donner à tous une longue expérience de travail et les faire sortir à 100 pour 100 avec des diplômes, il n'y aurait pas, pour autant, plus d'emplois à leur disposition. Le problème est structurel et on le sait très bien. On continue pourtant à fonder les réformes sur I'idée erronée que ce sont les individus qui sont responsables de leur situation de précarité et que la seule façon de se sortir de cette impasse est de développer l'entreprise qui, elle, va créer des emplois. Tout cela relève du mythe. Du reste, les emplois qui sont créés sont excessivement précaires et ne permettent aucunement de garantir l'élaboration de véritables projets de vie.

Un autre aspect important des changements de politiques concerne l'image des assistés sociaux. La crise économique a en effet frappé des gens qu'on avait pu croire à l'abri, jusque-là, du chômage et de l'assistance sociale. Soudainement, le chômeur et l'assisté social, ce sont le frère, la sœur, le fils, le père, l'oncle ou la cousine. $C^{\prime}$ est ainsi que les gens ont appris à se distancier un peu de leurs préjugés et à les remettre en question. Actuellement, on assiste toutefois à un retour à ces préjugés. L'assisté social et le chômeur sont vus, désormais, comme des individus vivant au crochet de la société. On tente de faire passer l'idée que ces personnes doivent mériter leurs prestations.

L'exclusion dans une société ne se limite jamais à l'exclusion économique; I'exclusion, c'est aussi une société qui te regarde, qui te pointe du doigt, qui te condamne. Cette façon de cibler les plus démunis, me confiaient des intervenantes en maison d'hébergement, se traduit par le renoncement des femmes violentées à quitter leur conjoint; elles préfèrent se résigner, $n^{\prime}$ ont $d^{\prime}$ autre choix que de demeurer avec le conjoint violent, parce que cette situation vaut mieux que l'opprobre d'être «sur l'aide sociale».

\footnotetext{
Finalement, si je comprends bien ce que vous dites, $c^{\prime}$ est que les changements de politiques sociales n'ont pas seulement eu un effet direct sur les possibilités matérielles et financières des femmes de
} 
s'affranchir des situations d'abus, de violence ou de situations tout simplement intenables, mais que les changements de politiques sociales ont aussi d'importantes répercussions sur le plan symbolique dans lequel on pourrait situer cet affranchissement?

Oui. Mais il y a aussi cette réalité qui fait que lorsqu'une femme n'est pas scolarisée, que, pour une raison ou une autre, elle a été longtemps en dehors du marché du travail, il ne lui est pas possible d'obtenir à court terme un emploi salarié ou, dans le meilleur des cas, elle a accès à un tel emploi mais pour une durée limitée seulement - le plus souvent au salaire minimum. Une fois que celui-ci sera terminé, une période de chômage s'enclenchera avec une rente basée sur ce faible salaire et, après cette période de prestation, ce qui I'attend c'est possiblement un séjour à l'aide sociale avec le phénomène d'endettement que cela suppose, etc. L'enchaînement de ces situations, voilà de quoi décourager n'importe quelle femme, même celle qui désire quitter un conjoint violent. II est donc sûr que dans la mesure où l'emploi salarié actuellement est bloqué, où les mesures sociales, les acquis sociaux sont de plus en plus grugés, les femmes retournent ou se maintiennent dans des situations de dépendance excessive. La dépendance, c'est la fragilité, c'est ce qui rend vulnérable.

En contrepartie, si cette femme pouvait bénéficier d'une aide sociale, avec un montant de base décent, sans se faire continuellement harceler pour qu'elle retourne à l'emploi, si elle avait accès à un logement convenable et à des services de garde le temps de réorganiser sa vie, la situation se présenterait sans doute fort différemment pour elle.

Ce qu'on veut faire actuellement avec les services de garde entre dans la même logique que la réforme des pensions de vieillesse dont j'ai parlé plus tôt. En fait, on veut réserver ces services pour les plus pauvres et faire payer les autres. Mais lorsque vous avez des revenus qui permettent à peine de surnager, ajouter un $50 \$$ ou un 100 \$ par mois suffit à débalancer entièrement le budget familial. Que feront alors ces familles? Elles retourneront à des mesures de bricolage avec la voisine et accrocheront une clé au cou de leur enfant en espérant que la vie soit clémente et les protège.

Je suis de la génération des femmes qui se sont battues pour les garderies. Je suis aussi de la génération des femmes qui ont eu des enfants à clé. Je suis de l'époque où, dès 3 heures de l'après-midi, je commençais à m'inquiéter. Je me demandais si mon enfant était chez la voisine, si tout se passait bien, si rien n'était arrivé... Aujourd'hui, 
je vois les possibilités qui sont en place et je trouve cela extraordinaire pour un parent de pouvoir aller reconduire son enfant à la garderie vers $7 \mathrm{~h}-7 \mathrm{~h} 30$ le matin et de le reprendre, en fin de journée, en sachant qu'entre-temps il est bien en sécurité. Sur le plan de la qualité de vie et d'esprit, c'est inestimable. Mais ces services, comme bien d'autres, n'entrent pas dans la logique du «basic needs ». Ces services sont vus comme un luxe.

\section{Lucie, nous avons beaucoup parlé de l'impact des changements de politiques sociales sur les conditions de vie des femmes et des personnes âgées qui, pour la plupart, bien sûr, sont aussi des femmes. Mais pensez-vous que ces changements de politiques ont également un impact sur les jeunes qui sont vus aujourd'hui comme les grands exclus de la société?}

Je suis heureuse que vous me posiez cette question et j'aimerais y répondre en prenant l'angle du non-accès à l'emploi et de ses répercussions sur l'identité masculine. En fait, de la même façon que les changements des politiques sociales s'articulent sur l'idée plus ou moins explicite que le maternage privé et social est une affaire de femmes, de la même façon on continue de compter sur les hommes en tant que pourvoyeurs et ce message est continuellement renforcé socialement. L'identité d'homme s'acquiert donc en très grande partie en ayant un emploi. Or, des emplois, il y en a de moins en moins et, Iorsqu'on en a un, celui-ci ne permet pas toujours d'acquérir une qualité de vie et des conditions valables. En d'autres mots, il ne s'agit pas d'emplois gratifiants.

Les gars vivent, à travers cette situation, une crise identitaire très profonde. Partout, c'est bloqué ; il n'y a pas de solution pour eux et ce désespoir est générateur de violence. Je ne voudrais pas avoir l'air de justifier la violence. La violence, quelle qu'elle soit, restera toujours un acte de domination envers les femmes et, dans ce contexte, les hommes ont à se positionner. Ce que je veux dire, en fait, c'est que le contexte économique et social que I'on vit actuellement rend les femmes encore plus vulnérables face à ces hommes qui, au fond, adoptent comme exutoire une position de dominant au sein de la famille. À mon avis, les situations de violence familiale vont devenir de plus en plus dramatiques et $c^{\prime}$ est pourquoi il faut repenser entièrement les paradigmes organisationnels de la société.

Plus que les autres, les jeunes sont vulnérables parce qu'il $\mathrm{n}^{\prime}$ existe aucune perspective d'avenir pour eux. Ils sont sans emploi indépendamment du fait qu'ils soient ou non scolarisés, et cela est 
très dramatique. On leur a dit "Qui s'instruit s'enrichit». On leur a dit que c'était d'abord et avant tout une question de mérite et d'efforts. Ce n'est pourtant pas l'effort qui a manqué. Nous avons à notre disposition une génération de jeunes instruits mais sans emploi qui, dans le meilleur des cas, se retrouvent avec une jobine, puis une autre, puis encore une autre. Puis à trente ans, toujours à la recherche d'un emploi, lorsqu'ils vont présenter leur C.V., tout ce qu'on trouve à leur dire c'est qu'ils sont pas mal instables...

Il y a quelque chose de profondément changé entre les gens de ma génération et les jeunes. Pour nous, I'emploi permanent était acquis; pour eux, le seul acquis, c'est qu'ils n'en n'auront jamais. II ne faut donc pas s'étonner que le travail salarié ne soit pas le centre de leur vie ou, encore, lorsqu'il est important, qu'il ne représente pas forcément toute leur vie. Pour eux, il est important d'aménager $\mathrm{d}^{\prime}$ autres espaces dans lesquels ils se sentent bien et dans lesquels ils se réalisent, parce qu'ils ne savent jamais à quel moment le travail salarié va leur glisser sous les pieds.

Dans un tel contexte, on aurait évidemment pu s'attendre à ce que les politiques sociales créent un mouvement de balancier et permettent aux individus d'avoir accès à un minimum de vie décente. Mais, au lieu de cela, on nous tire le tapis de sous les pieds et les femmes, comme les jeunes, à l'intérieur de ces nouvelles normes, sont particulièrement touchées. 